Pacific Journal of Mathematics

FACTORIZATION THEOREMS FOR DIFERENT CLASSES OF
ANALYTIC FUNCTIONS IN MULTIPLY CONNECTED
DOMAINS

DMITRY KHAVINSON 


\title{
FACTORIZATION THEOREMS FOR DIFFERENT CLASSES OF ANALYTIC FUNCTIONS IN MULTIPLY CONNECTED DOMAINS
}

\author{
D. KHAVINSON
}

This paper consists of four sections. In the first section we give a survey on the reproducing kernel for harmonic functions in finitely-connected Jordan regions. We also prove a certain version of Fatou's theorem which we will use in the next sections.

In the second part we construct the generalized Schwarz kernel for an arbitrary finitely-connected Jordan domain. This kernel reproduces any continuous single-valued analytic function inside the domain by the boundary values of its real part. Also, we give an explicit formula for the real part of this kernel in terms of the harmonic measures.

In the third section we study the Blaschke products in arbitrary Jordan domains.

The main results are contained in the fourth section. There we prove factorization theorems for the classes $N, N_{+}, H_{p}$ and $E_{p}$.

Introduction. It is well known that R. Nevanlinna's and V. I. Smirnov's factorization theorems have been very useful for many problems concerning analytic functions in the unit disc (e.g. see [8], [9], [15], [19]). Unfortunately, a direct attempt to extend these results to multiply connected domains has been unsuccessful. The fact is that the most natural function to play the role of the Schwarz kernel in such domains is not single-valued. Another problem appearing in that case is to define the Blaschke factor. It is obvious that even in an annulus one cannot find a single-valued function $f(z)$ satisfying the following properties: (1) $f(z)$ vanishes only at one given point; (2) $f(z)$ is continuous up to the boundary; (3) $|f(z)|$ is equal to 1 on the whole boundary of the annulus.

Many papers investigating the classes of analytic functions in multiply-connected domains have appeared. We refer the reader to the survey by S. Ya. Havinson and G. C. Tumarkin [10] which is quite detailed. The construction of the "Blaschke products" in finitely connected regions was suggested by V. A. Zmorovič. The convergence theorem for the products of that type has been proved by P. M. Tamrazov in [22].

The first attempts to generalize the Schwarz formula to finitely connected domains had already taken place in the 19th century. (By the 
"Schwarz formula" we understand the integral representation of an analytic function inside the domain in terms of the boundary values of its real part.)

The papers [24] and [1] contain many results obtained while trying to solve this problem. In [5-7] L. E. Dunduchenko and S. A. Kas'yanyuk have proved the factorization theorem for functions meromorphic in circular domains, belonging to the Nevanlinna class $N$ there and admitting only finitely many zeroes and poles inside the domain.

They used the analog of the Schwarz kernel which had been suggested by Zmorovič in [23] for the circular domains.

The most convenient construction of the Schwarz kernel for the solution of the factorization problem was given by R. Coifman and G. Weiss in [3]. In that paper they considered domains bounded by analytic curves. For those domains, they have proved the factorization theorem for the analytic functions of Nevanlinna's class $N$. Although the authors pointed out the possibility of extending their results to the classes $N_{+}$and $H_{p}$, they did not give explicit statements. Some further investigation in this direction is contained in T. S. Kuzina's paper [17].

If the boundary of the domain is only rectifiable (without assuming any smoothness conditions), or, furthermore, if it consists of arbitrary Jordan curves, the construction of the Schwarz kernel given in [3] cannot be applied. In this case, it is necessary to start out with the general reproducing kernel for harmonic functions. Generally speaking, this kernel is the quotient of the derivatives of the harmonic measures. Such a kernel has often been used in the theory of harmonic functions (see [18], [2]). To make its conjugate function single-valued we follow the procedure given in [3]. As a result, we obtain the factorization representations for the classes $N, N_{+}$and $H_{p}$ in arbitrary Jordan domains and for the classes $E_{p}$ in the domains with Jordan rectifiable boundaries.

\section{The generalized Green kernel and the Green-Stieltjes integral in} finitely connected domains. Let $G$ be a multiply-connected domain with the boundary $\Gamma$ consisting of $n$ disjoint Jordan curves $\gamma_{j} . z_{0}$ is a fixed point in $G . \omega(E, z, G)$ denotes the harmonic measure of the set $E \subset \Gamma$ taken at the point $z \in G$. Let $d \mu(\xi)$ be a finite real Baire measure on $\Gamma$. According to Koebe's theorem (see [9]) there exists a function $w=\psi(z)$ mapping $G$ conformally onto the circular domain $\mathscr{K}$. Note that $\psi(z)$ is bijective and continuous up to the boundary $\Gamma$. Let $z=\phi(w)=\psi^{-1}(w) . w_{0}=\psi\left(z_{0}\right)$. Let $g(z, \zeta), g(w, t)$ be the Green functions in $G$ and $\mathscr{K}$, respectively. 
Consider the following function on $\mathscr{K} \times \partial \mathscr{K}$ :

$$
\bar{K}(w, t)=\frac{1}{2 \pi} \frac{\partial g(w, t) / \partial n_{t}}{\partial g\left(w_{0}, t\right) / \partial n_{t}},
$$

where $w \in K, t \in \partial K$ and $\partial / \partial n_{t}$ is the derivative in the direction of the inner normal at the point $t$. Then $\bar{K}(w, t)$ is harmonic as a function of $w$ and continuous as a function of $t$. Note that the harmonic measure $d \omega(E, w, \mathscr{K})$ is absolutely continuous with respect to Lebesuge measure $d s$ on $\partial \mathcal{K}$, i.e.

$$
d \omega(E, w, \mathscr{K})=\frac{\partial}{\partial n_{t}} g(w, t) d s .
$$

Therefore, the function $\bar{K}(w, t)$ can also be defined as the Radon-Nikodym derivative at the point $t$ of the measure $(1 / 2 \pi) d \omega(E, w, \mathscr{K})$ with respect to the measure $d \omega\left(E, w_{0}, \mathscr{K}\right)$. Since harmonic measures are conformal invariants, then we can transfer the function $\bar{K}(w, t)$ into $G$ as follows: Let $z \in G, \zeta \in \Gamma, \psi(\zeta)=t, \psi(z)=w$. Then we define

$$
K(z, \zeta)=\left.\frac{1}{2 \pi} \frac{d \omega(E, z, G)}{d \omega\left(E, z_{0}, G\right)}\right|_{\zeta}=\bar{K}(w, t) .
$$

In other words, $K(z, \zeta)$ is the Radon-Nikodym derivative at the point $\zeta$ of the measure $(1 / 2 \pi) d \omega(E, z, G)$ with respect to the measure $d \omega\left(E, z_{0}, G\right)$. Note that in our case this derivative turns out to be a continuous function of $\zeta$. Also, (1.1) implies that $K(z, \zeta)$ is harmonic in $G$ as a function of $z$.

Consider the function $u(z)$ defined by

$$
u(z)=\int_{\Gamma} K(z, \zeta) d \mu(\zeta)
$$

It is clear that the function $u(z)$ is harmonic in $G$.

REMARK 1. Let $d \bar{\mu}(t)$ be the measure on $\partial K$ defined by $\bar{\mu}(E)=$ $\mu(\phi(E))$ for all Baire sets $E \subset \partial K$. Then by (1.1) we have

$$
u(z)=\int_{\Gamma} K(z, \zeta) d \mu(\zeta)=\int_{\partial K} \bar{K}(w, t) d \bar{\mu}(t)=u(\psi(z))
$$

We call the integrals (1.2) the Green-Stieltjes integrals. We also call $K(z, \zeta)$ the generalized Green kernel in $G$. 
If $u(z)$ can be represented in the form

$$
u(z)=\int_{\Gamma} K(z, \zeta) f(\zeta) d \omega\left(E, z_{0}, G\right),
$$

where $f(\zeta) \in L^{1}(d \omega)$, then we shall call $u(z)$ the Green-Lebesgue integral.

The following two theorems are well known (see $[18,2,11])$.

THEOREM 1.1. Let $z=\alpha(\tau)$ be the uniformization mapping of the unit disc $\mathscr{Q}$ onto $G$. $\mathcal{G}$ denotes the group of Möbius transformations of $\mathscr{D}$ corresponding to $\alpha(\tau)$. Let $u(z)$ be a harmonic function in $G$. Then the following statements are equivalent.

(1) $|u(z)|$ has a harmonic majorant in $G$.

(2) $u(z)=u^{1}(z)-u^{2}(z), u^{i}(z) \geq 0$ in $G, i=1,2$.

(3) The function $u^{*}(\tau)=u[\alpha(\tau)]$ is automorphic with respect to $\mathcal{G}$ and representable by the Poisson-Stieltjes integral in $\mathscr{D}$.

$$
u^{+}(z)=\left[\begin{array}{ll}
u(z), & u(z) \geq 0, \\
0, & u(z)<0,
\end{array} \text { has a harmonic majorant in } G .\right.
$$

(5) If $\left\{G^{i}\right\}_{1}^{\infty}$ is a sequence of domains such that $G^{i} \subset G^{i+1}, \cup_{i=1}^{\infty} G^{i}=G$ and $\omega^{i}\left(E, z_{0}, G^{i}\right)$ are the harmonic measures on $\partial G^{i}$, then

$$
\varlimsup_{i \rightarrow \infty} \int_{\partial G^{i}}|u| d \omega^{i}\left(E, z_{0}, G^{i}\right) \leq C_{u}<+\infty .
$$

(6)

$$
\varlimsup_{i \rightarrow \infty} \int_{\partial G^{i}} u^{+} d \omega^{i}\left(E, z_{0}, G^{i}\right) \leq C_{u}^{\prime}<+\infty .
$$

(7) $u(z)$ is representable by the Green-Stieltjes integral in $G$.

THeOREM 1.2. Let $u(z)$ be a harmonic function in $G$ and let $\alpha(\tau), \mathcal{G}$ be the same as in Theorem 1.1. Then the following statements are equivalent.

(1) The function $u^{*}(\tau)=u[\alpha(\tau)]$ is representable by the PoissonLebesgue integral in $\mathscr{Q}$ and $u^{*}(\tau)$ is automorphic with respect to the group $\mathcal{G}$.

(2) If the sequence $\left\{G^{i}\right\}^{\infty}$ is as in Theorem 1.1., then the integrals $\left\{\int_{\partial G^{i}}|u| d \omega^{i}\left(E, z_{0}, G^{i}\right)\right\}$ are uniformly absolutely continuous with respect to the harmonic measure ( $c f .[\mathbf{1 1}])$.

(3) $u(z)=u^{1}(z)-u^{2}(z)$ where $u^{i}(z) \geq 0, i=1,2$, and there exist nondecreasing sequences of bounded harmonic functions $\left\{u^{i}(z)\right\}_{n=1}^{\infty}$ converging uniformly to $u^{i}(z)$ on the compact subsets of $G$. 
(4) The least harmonic majorants $u^{M}(z)$ of the subharmonic functions $[|u(z)|-M]^{+}$converge to zero uniformly on the compact subsets of $G$ as $M \rightarrow \infty$.

(5) $u(z)$ is representable by the Green-Lebesgue integral (1.3).

Let us study the boundary behavior of the Green-Stieltjes integrals.

Let $\zeta \in \Gamma$ and let $\gamma_{\zeta}$ be a Jordan half-open arc such that $\gamma_{\zeta} \in G$ and $\zeta$ is its endpoint. Let $f(z)$ be any function in $G$. Assume there exists the limit $A=\lim _{z \rightarrow \zeta ; z \in \gamma_{\zeta}} f(z)$ (finite or infinite). Then following [4] we call $A$ the asymptotic value of $f(z)$ at the point $\zeta$ along $\gamma_{\zeta}$. If $f(z)$ has the asymptotic value $A$ at $\zeta$ along a certain curve $\gamma_{\zeta}^{0}$ (it is possible that $A= \pm \infty$ ) and the asymptotic value along any other curve $\gamma_{\zeta}$ is either equal to $A$ or does not exist, then $A$ is called the asymptotic boundary value of $f(z)$ at $\zeta$. According to the celebrated result of F. Bagemihl, an arbitrary function $f(z)$ has asymptotic boundary values at all points $\zeta \in \Gamma$ except a certain countable set (see [4]). Let us fix arbitrary points $\zeta_{i} \in \gamma_{i}$, $i=1, \ldots, n$. Define the generating function $\tilde{\mu}$ for the measure $d \mu$ as follows. Let $\zeta \in \gamma_{l}$ and $\widehat{\zeta_{i} \zeta}$ denote the arc of $\gamma_{l}$ between $\zeta_{i}$ and $\zeta$ oriented in the same directions as $\gamma_{i}$. Then we set

$$
\tilde{\mu}\left(\omega\left(\widehat{\zeta_{i} \zeta}, z_{0}, G\right)\right)=\mu\left(\widehat{\zeta_{i} \zeta}\right) .
$$

It is clear that $\tilde{\mu}$ has bounded variation as a function of the parameter $\omega\left(\overparen{\zeta_{l} \zeta}, z_{0}, G\right)$.

THEOREM 1.3 ( $P$. Fatou's theorem). Let us consider all points $\zeta_{0} \in \gamma_{i}$, $i=1, \ldots, n$, such that there exists a finite or infinite derivative

$$
\tilde{\mu}^{\prime}\left(\zeta_{0}\right)=\lim _{\zeta \rightarrow \zeta_{0}} \frac{\tilde{\mu}\left(\omega\left(\widehat{\zeta_{0} \zeta}, z_{0}, G\right)\right)}{\omega\left(\widehat{\zeta_{0} \zeta}, z_{0}, G\right)} .
$$

Then the integral (1.2) has asymptotic boundary values $\tilde{\mu}^{\prime}\left(\zeta_{0}\right)$ at all such points $\zeta_{0}$ except, maybe, a countable set.

Proof. According to the invariance of harmonic measures and Remark 1 it suffices to prove our theorem for the circular domain $\mathscr{K}$. At first, we note that if $u(w)$ is representable by the integral (1.2) in $\mathscr{K}$ with the measure $d \bar{\mu}$, then there exists a Baire measure $d \nu$ on $\partial \mathcal{K}$ such that

$$
u(w)=\frac{1}{2 \pi} \int_{\partial K} \frac{\partial g(w, t)}{\partial n_{t}} d \nu(t) .
$$


In reality, the analyticity of $\partial \mathcal{K}$ implies that $\partial g(w, t) / \partial n_{t}$ is harmonic in the neighborhood of $\partial K$ and does not vanish in that neighborhood. Then setting

$$
\nu(E)=\int_{E \cap \partial K} \frac{1}{\partial g\left(w_{0}, t\right) / \partial n_{t}} d \bar{\mu}
$$

for any Baire set $E \subset \mathbf{R}^{2}$, we obtain (1.4). Let $\partial \mathscr{K}=\cup_{j=1}^{n} l_{j}$, where $l_{j}$ are circles. Let $\nu(t)$ be the generating function of the measure $d \nu$. Fix $i$. Let $t_{0} \in l_{i}$. Assume there exists a finite or infinite derivative $\nu^{\prime}\left(t_{0}\right)$. Let $w$ tend to $t_{0}$. Then $\partial g(w, t) / \partial n_{t} \rightarrow 0$ uniformly on all $l_{j}, j \neq i$. Hence,

$$
u(w)=\frac{1}{2 \pi} \int_{l_{i}} \frac{\partial}{\partial n_{t}} g(w, t) d \nu(t)+o(1)
$$

if $w$ is sufficiently close to $t_{0}$. Let $g_{i}(w, t)$ be the Green function of the simply-connected domain $\mathcal{K}_{i}$ bounded by $l_{i}$ and such that $\mathcal{K}_{i} \ni \mathcal{K}$. We define the function $r_{w}(t)$ as follows:

$$
r_{w}(t)=g(w, t)-g_{i}(w, t) .
$$

$r_{w}(t)$ is harmonic in $\mathscr{K}$. As $w \rightarrow t_{0}, r_{w}(t) \rightarrow 0$ uniformly outside a certain neighborhood of $t_{0}$. Since $r_{w}(t) \equiv 0$ on $l_{i}$, then according to the Riemann-Schwarz symmetry principle we can continue $r_{w}(t)$ through $l_{i}$. Therefore, $r_{w}(t) \rightarrow 0$ as $w \rightarrow t_{0}$ uniformly on all the circles $l_{j}^{\prime}$ symmetric to $l_{j}, j \neq i$, with respect to $l_{i}$. Hence, $r_{w}(t) \rightarrow 0$ as $w \rightarrow t_{0}$ uniformly in the whole domain bounded by $l_{j}, l_{j}^{\prime}, j \neq i$. Then $\partial r_{w}(t) / \partial n_{t} \rightarrow 0$ uniformly as $w \rightarrow t_{0}$. But $\partial g_{i}(w, t) / \partial n_{t}, t \in \partial \mathcal{K}_{i}$ is the classical Poisson kernel in $\mathcal{K}_{i}$. Thus, from (1.5) we obtain that

$$
u(w)=\frac{1}{2 \pi} \int_{l_{i}} \frac{\partial}{\partial n_{t}} g_{i}(w, t) d \nu(t)+o(1) .
$$

Assume $w \rightarrow t_{0}$ inside a certain angle in $\mathcal{K}$. Then from (1.6) and the classical Fatou theorem (see $[8,9,15,19])$ we obtain that $u(w) \rightarrow \nu^{\prime}\left(t_{0}\right)$. Since

$$
\frac{d \bar{\mu}}{d \omega}=\frac{d \bar{\mu}}{d \nu} \cdot \frac{d \nu}{d \omega}=\frac{\partial}{\partial n_{t}} g\left(w_{0}, t\right) \cdot \frac{d \nu}{\left(\partial g\left(w_{0}, t\right) / \partial n_{t}\right) d t}=\nu^{\prime}\left(t_{0}\right),
$$

then $u(w) \rightarrow \bar{\mu}^{\prime}\left(t_{0}\right)$ as $w \rightarrow t_{0}$ inside any angle in $\mathscr{K}$. Applying the abovementioned theorem of Bagemihl, we complete the proof.

COROLlaRY 1.1. The integrals (1.2) have finite asymptotic boundary values almost everywhere with respect to harmonic measure. 
COROLlaRY 1.2. The representing measure $d \mu$ for the harmonic function $u(z)$ in (1.2) is unique.

Proof. Let us assume there are two measures $d \mu_{1}, d \mu_{2}$ such that

$$
u(z)=\int_{\Gamma} K(z, \zeta) d \mu_{1}=\int_{\Gamma} K(z, \zeta) d \mu_{2} .
$$

Let $d \sigma=d \mu_{1}-d \mu_{2}$. Then

$$
v(z)=\int_{\Gamma} K(z, \zeta) d \sigma(\zeta) \equiv 0 .
$$

According to Theorem 1.3 we obtain from (1.7) that $d \sigma$ is singular with respect to $d \omega$. Then, according to the De La Vallee-Poisson theorem, $|d \sigma / d \omega|=\infty$ a.e. with respect to $d \sigma$ (see [21]). In the proof of Theorem 1.3 we have actually shown that at each point where there exists $d \sigma / d \omega$, there always exists an asymptotic value of $v(z)$ equal to $d \sigma / d \omega$. So, a.e. with respect to $d \sigma$ there exist asymptotic values of $v(z)$ equal to $\pm \infty$. This contradicts (1.7).

For the sake of completeness we state and prove the decomposition theorem for Green-Stieltjes integrals. A similar result for Green-Lebesgue integrals was proved in [11].

The following lemma is known (see [12], [14]).

LEMMA 1.1. Let $\left\{G^{i}\right\}_{1}^{\infty}$ be a sequence of domains with smooth boundaries such that $\cup_{i=1}^{\infty} G^{i}=G, G^{i} \subset G^{i+1}, \partial G^{i}=\Gamma^{i}=\cup_{i=1}^{n} \gamma_{j}^{i}$, where $\gamma_{j}^{i}$ is homologous to $\gamma_{j} \subset \Gamma$. Let $G_{j}^{i}$ denote the domain bounded by $\gamma_{j}^{l}$ such that $G_{j}^{i} \supset G^{i}$. Let $g^{i}(z, \zeta), g_{j}^{i}(z, \zeta)$ be the Green functions of $G^{i}$ and $G_{j}^{i}$, respectively. Then there exists a constant $\lambda$ such that for all $i$,

$$
1 \leq \frac{\partial g_{j}^{i}\left(z, z_{0}\right) / \partial n_{z}}{\partial g^{i}\left(z, z_{0}\right) / \partial n_{z}} \leq \lambda, \quad z \in \gamma_{j}^{i}, j=1, \ldots, n .
$$

THEOREM 1.4. Let $u(z)$ be representable by the integral (1.2). Let $G_{j}$ be a domain bounded by $\gamma_{j}$ such that $G_{j} \supset G$. Fix arbitrary $\alpha_{j} \in \overline{\mathbf{C}} \backslash G_{j}$, $j=1, \ldots, n$. Then

$$
u(z)=\sum_{1}^{n} u_{j}(z)
$$

where

$$
u_{j}(z)=c_{J} \ln \left|z-\alpha_{j}\right|+u_{j}^{\prime}(z)
$$


$c_{j}$ are constants depending only on $u(z)$. If $\infty \notin G_{j}$, then $c_{j}=0$. For each $j=1, \ldots, n u_{j}^{\prime}(z)$ is harmonic in $G_{j}$. Moreover, each $u_{j}^{\prime}(z)$ is representable by the Green-Stieltjes integral in $G_{j}, u_{j}^{\prime}(\infty)=0$. The functions $u_{j}^{\prime}(z)$, $j=1, \ldots, n$, depend only on $u(z)$ and the choice of $\alpha_{j}$.

Proof. Let the sequence $\left\{G^{i}\right\}_{1}^{\infty}$ be the same as in Lemma 1.1. According to Green's theorem applied to $G^{i}$, we obtain

$$
\begin{aligned}
u(z) & =\frac{1}{2 \pi} \int_{\Gamma^{i}} u(\zeta)\left[\frac{\partial}{\partial n} \ln \frac{1}{|z-\zeta|}\right] d s-\frac{1}{2 \pi} \int_{\Gamma^{i}} \ln \frac{1}{|z-\zeta|} \frac{\partial u}{\partial n} d s \\
& =\sum_{j=1}^{n} u_{j}(z), \quad z \in G^{i},
\end{aligned}
$$

where

$$
\begin{aligned}
u_{j}(z)= & \frac{1}{2 \pi} \int_{\gamma_{j}^{i}} u(\zeta)\left[\frac{\partial}{\partial n} \ln \frac{1}{|z-\zeta|}\right] d s \\
& -\frac{1}{2 \pi} \int_{\gamma_{j}^{i}} \ln \frac{1}{|z-\zeta|} \frac{\partial u}{\partial n} d s .
\end{aligned}
$$

It is clear that the $u_{j}(z), j=1, \ldots, n$, do not depend on $i$. Also, they are harmonic in $G_{j}$, respectively, except, maybe, at infinity (if $\infty \in G_{j}$ ). In a neighborhood of $\infty$ we have

$$
u_{j}(z)=c_{j} \ln \left|z-\alpha_{j}\right|+u_{j}^{\prime}(z), \quad j=1, \ldots, n,
$$

where

$$
c_{j}=\frac{1}{2 \pi} \int_{\gamma_{j}^{j}} \frac{\partial u}{\partial n} d s, \quad j=1, \ldots, n
$$

and $u_{j}^{\prime}(z)$ are harmonic at $\infty, j=1, \ldots, n$. Fix $j$. According to (1.9) and (1.10) there exists the constant $M$ such that the inequality

$$
\left|u_{j}^{\prime}(z)\right| \leq|u(z)|+M
$$

holds near $\gamma_{j}^{i}$. Let $d \omega^{i}\left(E, z_{0}, G^{i}\right), d \omega_{j}^{i}\left(E, z_{0}, G_{j}^{i}\right)$ be the harmonic measures on $\partial G^{i}$ and $\partial G_{j}^{i}$, respectively. Then, by Lemma 1.1, we have

$$
1 \leq \frac{d \omega_{j}^{i}\left(E, z_{0}, G_{j}^{i}\right)}{d \omega^{i}\left(E, z_{0}, G^{i}\right)} \leq \lambda .
$$


Since $u(z)$ is representable by the integral (1.2) in $G$, then applying p. 5 of Theorem 1.1 and estimates (1.11) and (1.12), we obtain

$$
\int_{\gamma_{j}^{\prime}}\left|u_{j}^{\prime}\right| d \omega_{j}^{i}\left(E, z_{0}, G_{j}^{i}\right) \leq \lambda \int_{\gamma_{j}^{\prime}}|u| d \omega^{i}\left(E, z_{0}, G^{i}\right)+M \leq \lambda C_{u}+M<+\infty
$$

Then applying Theorem 1.1 again we obtain that each $u_{j}^{\prime}(z)$ is representable by the integral (1.2) in $G_{j}$.

In $\S 4$ we will make use of the following.

THEOREM 1.5. Let $u(z)$ be a harmonic function in $G .\left\{G^{i}\right\}_{1}^{\infty}$, $d \omega^{i}\left(E, z_{0}, G^{i}\right)$ are the same as above. The following statements are equivalent.

(1) The integrals $\left\{\int_{\partial G^{i}} u^{+}(\zeta) d \omega^{i}\left(E, z_{0}, G^{i}\right)\right\}$ are uniformly absolutely continuous with respect to the harmonic measure.

(2) $u(z)$ is representable by the integral (1.2) with the measure $d \mu(\zeta)=$ $F(\zeta) d \omega+d \nu$, where $d \nu \leq 0$ and $d \nu$ is singular with respect to $d \omega . F(\zeta)$ is equal to the asymptotic boundary values of $u(z)$ a.e. with respect to $d \omega$ on $\Gamma$.

(3) $u(z)$ is representable by the integral (1.2) with the measure $d \mu(\zeta)=$ $\Psi(\zeta) d \omega+d \nu_{1}$, where $d \nu_{1} \leq 0, \Psi(\zeta) \geq 0$ and $\Psi(\zeta)$ is equal to the asymptotic boundry values of $u^{+}(z)$ a.e. with respect to $d \omega$.

REMARK 2. The class of harmonic functions characterized by Theorem 1.5 as well as the corresponding class of subharmonic functions was introduced by I. I. Privalov in [20] for the unit disc. In [16] these classes were investigated in simply connected domains with rectifiable boundaries.

$$
\begin{aligned}
& \text { Proof. (2) } \Rightarrow(3) \text {. Let } F(\zeta)=F^{+}(\zeta)-F^{-}(\zeta) \text {. Then } \\
& \qquad d \mu=F^{+}(\zeta) d \omega+d \nu-F^{-}(\zeta) d \omega=F^{+}(\zeta) d \omega+d \nu_{1},
\end{aligned}
$$

where $d \nu_{1} \leq 0$.

(3) $\Rightarrow(2)$. According to the Lebesgue decomposition, we have $d \nu_{1}=$ $f(\zeta) d \omega+d \nu$, where $d \nu$ is singular. Since $d \nu_{1} \leq 0$, then $f(\zeta) \leq 0$ and $d \nu \leq 0$. Therefore,

$$
d \mu(\zeta)=\Psi(\zeta) d \omega+f(\zeta) d \omega+d \nu=F(\zeta) d \omega+d \nu .
$$

$(2) \Rightarrow(1)$. Since

$$
u(z)=\int_{\Gamma} K(z, \zeta) d \mu(\zeta) \leq \int_{\Gamma} K(z, \zeta) F^{+}(\zeta) d \omega=v(z),
$$


then $u^{+}(z) \leq v(z)$. According to Theorem 1.2 the integrals $\left\{\int_{\Gamma^{i}} v d \omega^{i}\right\}$ are uniformly absolutely continuous. Then the integrals $\left\{\int_{\Gamma^{i}} u^{+} d \omega^{i}\right\}$ are also uniformly absolutely continuous. In a similar way one can show that (1) $\Rightarrow(2)$. The theorem is proved.

\section{The Green-Schwarz kernel in finitely connected Jordan domains.} We keep the same notation as in $\S 1$. Let $\tilde{K}(z, \zeta)$ be the conjugate harmonic function of $K(z, \zeta)$ as $\zeta \in \Gamma$ is fixed. Let $f(z)=u(z)+i v(z)$ be a single-valued analytic function in $G$ such that $u(z)$ is continuous in $\bar{G}$. Then, according to Theorem 1.2 , we have

$$
u(z)=\int_{\Gamma} K(z, \zeta) u(\zeta) d \omega\left(E, z_{0}, G\right) .
$$

Set $p(z, \zeta)=K(z, \zeta)+i \tilde{K}(z, \zeta)$. Then

$$
f(z)=\int_{\Gamma} u(\zeta) p(z, \zeta) d \omega\left(E, z_{0}, G\right) .
$$

But if $u(\zeta)$ is an arbitrary real-valued continuous functions on $\Gamma$, then the integral (2.1) gives, in general, a multi-valued function in $G$. We want to construct the kernel $\mathscr{P}(z, \zeta)$ such that for any continuous function $\bar{u}(\zeta)$ on $\Gamma$ the function

$$
f(z)=\int_{\Gamma} \mathscr{P}(z, \zeta) \bar{u}(\zeta) d \omega\left(E, z_{0}, G\right)
$$

is analytic and single-valued in $G$. In the following construction we use the basic ideas of [3].

Let $\Gamma^{\prime}=\cup_{k=1}^{n} \gamma_{k}^{\prime}$, where $\gamma_{k}^{\prime}$ are analytic curves in $G$ and $\gamma_{k}^{\prime}$ is homologous to $\gamma_{k}, k=1, \ldots, n$. Let $G^{\prime} \subset G$ be a domain bounded by $\Gamma^{\prime}$. Let $\omega_{k}(z)$ be a harmonic measure of $\gamma_{k}, k=1, \ldots, n$, i.e. $\left.\omega_{k}\right|_{\gamma_{k}} \equiv 1$, $\left.\omega_{k}\right|_{\gamma_{j}} \equiv 0, j \neq k$, and $\omega_{k}$ is harmonic in $G$. $\omega_{k}^{\prime}$ are harmonic measures of $\gamma_{k}^{\prime}$ with respect to $G^{\prime}, k=1, \ldots, n$.

Let $u(z)$ be a harmonic function in $G$ and let $v(z)$ be its conjugate. According to the Cauchy-Riemann equations and Green's formula the conditions for $v(z)$ to be single-valued in $G$ can be written in the following form:

$$
\Delta_{\gamma_{k}} v=\int_{\gamma_{k}^{\prime}} \frac{\partial v}{\partial s} d s=-\int_{\gamma_{k}^{\prime}} \frac{\partial u}{\partial n} d s=-\int_{\Gamma^{\prime}} u \frac{\partial \omega_{k}^{\prime}}{\partial n} d s=0, \quad k=1, \ldots, n-1 .
$$

From now on we assume $\gamma_{1}, \ldots, \gamma_{n-1}$ lie inside of $\gamma_{n}$. Let

$$
\pi_{k j}=\int_{\gamma_{j}^{\prime}} \frac{\partial \tilde{\omega}_{k}}{\partial s} d s=-\int_{\gamma_{j}^{\prime}} \frac{\partial \omega_{k}}{\partial n} d s, \quad k, j=1, \ldots, n-1 .
$$


Here, $\tilde{\omega}_{k}$ is the conjugate function of $\omega_{k}$. As it is known (see [3]), the matrix $\left\|\pi_{k j}\right\|_{1}^{j-1}$ is symmetric and positive definite. Hence, $\operatorname{det}\left\|\pi_{k j}\right\| \neq 0$. Therefore, for any harmonic function $u(z)$ in $G$ there exist real numbers $\lambda_{1}, \ldots, \lambda_{n-1}$ such that the conjugate function of $u(z)-\sum_{1}^{n-1} \lambda_{j} \omega_{j}(z)$ is single-valued in $G$. Those $\lambda_{1}, \ldots, \lambda_{n-1}$ are uniquely defined by $u(z)$. By (2.2) and (2.3) it is also clear that $\lambda_{j}, j=1, \ldots, n-1$, satisfy the following equations:

$$
\sum_{j=1}^{n-1} \lambda_{j} \pi_{j K}=-\int_{\gamma_{k}^{\prime}} \frac{\partial u}{\partial n} d s, \quad k=1, \ldots, n-1 .
$$

THEOREM 2.1. There exists a unique function $\mathscr{P}(z, \zeta)$ continuous on $G \times \Gamma$ and satisfying the following properties: (1) If $\zeta \in \Gamma$ is fixed, then $\mathscr{P}(z, \zeta)$ is single-valued and analytic in $G$. (2) If $z \in G$ is fixed, then

$$
\begin{gathered}
\int_{\gamma_{k}} \mathscr{P}(z, \zeta) d \omega\left(E, z_{0}, G\right)=0, \quad k=1, \ldots, n-1 ; \\
\int_{\gamma_{n}} \mathscr{P}(z, \zeta) d \omega\left(E, z_{0}, G\right)=1 .
\end{gathered}
$$

(3) If $f(z)=u(z)+i v(z)$ is a single-valued analytic function in $G$ and $u(z)$ is continuous in $\bar{G}$, then

$$
f(z)=\int_{\Gamma} \mathcal{P}(z, \zeta) u(\zeta) d \omega\left(E, z_{0}, G\right)+i v\left(z_{0}\right) .
$$

We shall call $\mathscr{P}(z, \zeta)$ the Green-Schwarz kernel in $G$.

Proof. (1) Fix $\zeta \in \Gamma$. Choose the numbers $\Lambda_{1}(\zeta), \ldots, \Lambda_{n-1}(\zeta)$ such that the conjugate function $\tilde{R}(z, \zeta)$ of the function

$$
R(z, \zeta)=K(z, \zeta)-\sum_{1}^{n-1} \Lambda_{j}(\zeta) \omega_{j}(z)
$$

is single-valued in $G$ and $\tilde{R}\left(z_{0}, \zeta\right)=0$. In case of $u(z)=K(z, \zeta)$, from (2.4) it follows that $\Lambda_{1}(\zeta), \ldots, \Lambda_{n-1}(\zeta)$ are continuous functions of $\zeta$. Setting $\mathcal{P}(z, \zeta)=R(z, \zeta)+i \tilde{R}(z, \zeta)$, we complete the proof of (1).

(2) Let $\bar{u}(\zeta)$ be an arbitrary real-valued continuous function on $\Gamma$. Consider the following integrals:

$$
\begin{aligned}
& u(z)=\int_{\Gamma} K(z, \zeta) \bar{u}(\zeta) d \omega\left(E, z_{0}, G\right) \\
& u_{1}(z)=\int_{\Gamma} R(z, \zeta) \bar{u}(\zeta) d \omega\left(E, z_{0}, G\right)=u(z)-\sum_{1}^{n-1} \lambda_{j} \omega_{j}(z)
\end{aligned}
$$


where

$$
\begin{gathered}
\lambda_{j}=\int_{\Gamma} \Lambda_{j}(\zeta) \bar{u}(\zeta) d \omega\left(E, z_{0}, G\right) \\
\mathscr{F}(z)=\int_{\Gamma} \mathcal{P}(z, \zeta) \bar{u}(\zeta) d \omega\left(E, z_{0}, G\right)=u_{1}(z)+i v_{1}(z),
\end{gathered}
$$

where $v_{1}$ is the conjugate of $u_{1}$. Then $\mathscr{F}(z)$ is single-valued. Take $\bar{u}(\zeta)=$ $\omega_{n}(\zeta)$. Then $u(z)=\omega_{n}(z)$. Put $\lambda_{1}=\cdots=\lambda_{n-1}=-1$. Since system (2.4) has a unique solution, $u_{1}(z) \equiv 1$. Hence,

$$
\int_{\Gamma} \mathcal{P}(z, \zeta) \omega_{n}(\zeta) d \omega\left(E, z_{0}, G\right)=\int_{\gamma_{n}} \mathscr{P}(z, \zeta) d \omega\left(E, z_{0}, G\right) \equiv 1+i c
$$

But $\tilde{R}\left(z_{0}, \zeta\right)=0$. So $c=0$. Letting $\bar{u}(\zeta)=\omega_{k}(\zeta), k<n$, and putting $\lambda_{1}=0, \ldots, \lambda_{k}=-1, \lambda_{k+1}=0, \ldots, \lambda_{n-1}=0$, one can easily verify that

$$
\int_{\gamma_{k}} \mathscr{P}(z, \zeta) d \omega\left(E, z_{0}, G\right)=0
$$

(3) Let $f(z)=u(z)+i v(z)$ satisfy our hypothesis. Then system (2.4) has only a trivial solution. If we plug $\bar{u}(\zeta)=u(\zeta)$ into (2.5)-(2.7), then we obtain that $u_{1}(z) \equiv u(z)$ and $f(z)=\mathscr{F}(z)+i v\left(z_{0}\right)$.

To prove the uniqueness of $\mathcal{P}(z, \zeta)$, let us assume there exists another function $\mathscr{P}_{1}(z, \zeta)$ satisfying (1)-(3). Take an arbitrary real-valued function $\bar{u}(\zeta)$ continuous on $\Gamma$. Define $u, u_{1}$ and $\mathscr{F}$ by (2.5), (2.6) and (2.7), respectively. According to (2) and (3), we have

$$
\begin{aligned}
\mathscr{F}(z) & =\int_{\Gamma} \mathscr{P}_{1}(z, \zeta) u_{1}(\zeta) d \omega=\int_{\Gamma} \mathscr{P}_{1}(z, \zeta)\left(\bar{u}(\zeta)-\sum_{1}^{n-1} \lambda_{j} \omega_{j}(\zeta)\right) d \omega \\
& =\int_{\Gamma} \mathscr{P}_{1}(z, \zeta) \bar{u}(\zeta) d \omega=\int_{\Gamma} \mathscr{P}(z, \zeta) \bar{u}(\zeta) d \omega
\end{aligned}
$$

for all $z \in G$. Since $\bar{u}(\zeta)$ was an arbitrary continuous function, we obtain $\mathscr{P}_{1}(z, \zeta) \equiv \mathscr{P}(z, \zeta)$. The proof is complete.

To compute $\operatorname{Re} \mathscr{P}(z, \zeta)$ we have to introduce more notation.

At first, let us consider the circular domain $\mathcal{K}$. Let $\partial \mathcal{K}=\cup_{j=1}^{n} l_{j}$, and let $\omega_{j}$ be a harmonic measure of $l_{j}$. We define the functions $S_{j}(t)$ on aK as follows:

$$
S_{j}\left(t, w_{0}\right)=S_{j}(t)=\frac{\partial \omega_{j}(t) / \partial n_{t}}{\partial g\left(t, w_{0}\right) / \partial n_{t}}, \quad t \in \mathscr{K} ; j=1, \ldots, n-1 .
$$


It is clear that $S_{j}(t)$ are continuous functions on $\Gamma$. Let $\Gamma^{\prime}=\cup_{k=1}^{n} l_{k}^{\prime}$ be defined in the same way as above. Let $u(z)$ be a harmonic function in $\mathscr{K}$ continuous in $\overline{\mathcal{K}}$. Let $v(z)$ be its conjugate. Then, according to (2.2), we have

$$
\Delta_{l_{k}} v=-\int_{\Gamma^{\prime}} u \frac{\partial \omega_{k}^{\prime}}{\partial n} d s=-\int_{\Gamma^{\prime}} u(\tau) \frac{\partial \omega_{k}^{\prime}(\tau) / \partial n_{\tau}}{\partial g\left(t, w_{0}\right) / \partial n_{\tau}} \cdot \frac{\partial}{\partial n_{\tau}} g\left(\tau, w_{0}\right) d s .
$$

As $\Gamma^{\prime} \rightarrow \partial \mathscr{K}$ it can be easily seen that

$$
\int_{\Gamma^{\prime}} u(\tau) \frac{\partial \omega_{k}^{\prime}(\tau) / \partial n_{\tau}}{\partial g\left(\tau, w_{0}\right) / \partial n_{\tau}} \cdot \frac{\partial}{\partial n_{\tau}} g\left(\tau, w_{0}\right) d s \rightarrow \int_{\partial \mathscr{K}} \bar{u}(t) S_{k}(t) d \omega .
$$

Therefore,

$$
\Delta_{\gamma_{k}} v=-\int_{\partial \mathcal{K}} u(t) S_{k}(t) d \omega\left(E, w_{0}, \mathcal{H}\right) .
$$

Let $G$ be an arbitrary $n$-connected Jordan domain. $w=\psi(z)$ is as in $\S 1$. We put

$$
S_{J}\left(\zeta, z_{0}\right)=S_{J}(\zeta)=S_{j}(\psi(\zeta)), \quad \zeta \in \Gamma .
$$

Since both sides in (2.8) are invariant with respect to conformal maps, then (2.8) still holds for $G(u(z)=u(\psi(z))$, i.e.

$$
\Delta_{\gamma_{k}} v=-\int_{\Gamma} u(\zeta) S_{k}(\zeta) d \omega\left(E, z_{0}, G\right) .
$$

THEOREM 2.2. Let $\left\|q_{j k}\right\|_{1}^{n-1}=\left(\left\|\pi_{j k}\right\|_{1}^{n-1}\right)^{-1}$. Let $\mathcal{P}(z, \zeta)$ be the GreenSchwarz kernel in $G$. Then

$$
\operatorname{Re} \mathscr{P}(z, \zeta) \stackrel{\text { def }}{=} R(z, \zeta)=K(z, \zeta)-\sum_{j=1}^{n-1} \Lambda_{j}(\zeta) \omega_{j}(z)
$$

where

$$
\Lambda_{j}(\zeta)=-\sum_{k=1}^{n-1} q_{j k} S_{k}(\zeta)
$$

Proof. Let $\bar{u}(\zeta)$ be an arbitrary real-valued continuous function on $\Gamma$. Define $u(z)$ in $G$ by (2.5). From (2.4) and (2.9) we obtain

$$
\lambda_{J}=-\sum_{k=1}^{n-1} q_{j k} \int_{\Gamma} S_{k}(\zeta) \bar{u}(\zeta) d \omega\left(E, z_{0}, G\right) .
$$

At the same time, by (2.6), we have

$$
\lambda_{j}=\int_{\Gamma} \Lambda_{j}(\zeta) \bar{u}(\zeta) d \omega\left(E, z_{0}, G\right)
$$


Therefore,

$$
\int_{\Gamma}\left[\Lambda_{j}(\zeta)+\sum_{k=1}^{n-1} q_{j k} S_{k}(\zeta)\right] \bar{u}(\zeta) d \omega\left(E, z_{0}, G\right) \equiv 0
$$

for any $\bar{u}$. From this (2.10) follows.

3. The generalized Blaschke products. In the following theorem we put together the necessary information (most of which is known) concerning zeroes of analytic functions. We keep the same notation as in $\S \S 1,2$.

THEOREM 3.1. Let $f(z)$ be a single-valued analytic function in $G$. Let $\left\{z_{k}\right\}_{1}^{\infty}$ be the sequence of its zeroes in $G$. The following statements are equivalent:

(1) $\ln |f(z)|$ has a harmonic majorant in $G$.

(2) The series $\Sigma_{1}^{\infty} g\left(z_{k}, z\right)$ converges uniformly on compact subsets of $G \backslash\left\{z_{k}\right\}_{1}^{\infty}$.

(3) Let $\left\{z_{k}^{j}\right\}_{k=1}^{\infty}, j=1, \ldots, n$, be the subsequences of $\left\{z_{k}\right\}_{k=1}^{\infty}$ such that all cluster points of $\left\{z_{k}^{j}\right\}$ belong to $\gamma_{j} ;\left\{z_{k}\right\}_{1}^{\infty}=\bigcup_{j=1}^{n}\left\{z_{k}^{j}\right\}_{k=1}^{\infty},\left\{z_{k}^{i}\right\}_{k=1}^{\infty} \cap$ $\left\{z_{k}^{j}\right\}_{k=1}^{\infty}=\varnothing$ as $i \neq j$. Then the series $\sum_{k=1}^{\infty} g_{j}\left(z_{k}^{j}, z\right), j=1, \ldots, n$, converge uniformly on the compact subsets of $G_{j} \backslash\left\{z_{k}^{j}\right\}_{k=1}^{\infty}$.

(4) The series $\sum_{k=1}^{\infty}\left|\omega_{i}\left(z_{k}^{j}\right)-\delta_{i j}\right|, i=1, \ldots, n, j=1, \ldots, n$, converge. $\left(\delta_{i j}\right.$ is the Kronecker symbol.)

If $\Gamma=\cup_{j=1}^{n} \gamma_{j}$ is analytic and $\zeta_{j}$ denotes the closest point to $z_{j}$ on $\Gamma$, then (1)-(4) are equivalent to the following:

$$
\sum_{j=1}^{\infty}\left|z_{j}-\zeta_{j}\right|<+\infty
$$

Proof (1) $\Leftrightarrow$ (2) follows immediately from the Green-Jensen formula.

(2) $\Leftrightarrow(3)$. From Lemma 1.1 it easily follows that the following inequalities hold near each $\gamma_{j}, j=1, \ldots, n$ :

$$
1 \leq g_{j}\left(z, z_{0}\right) / g\left(z, z_{0}\right) \leq C_{j},
$$

where $C_{j}$ are certain constants. This implies that $(2) \Leftrightarrow(3)$.

$(2) \Leftrightarrow(4)$. Let us transfer everything into the circular domain $\mathcal{H}$. Let $g_{j}(w, t)$ be the Green functions of $\mathcal{K}_{j}, j=1, \ldots, n$, and $w_{k}=\psi\left(z_{k}\right)$. According to the invariance of Green's functions we have

$$
\sum_{1}^{\infty} g\left(z_{k}, z_{0}\right)=\sum_{1}^{\infty} g\left(w_{k}, w_{0}\right) .
$$


Since we have already proved that $(2) \Leftrightarrow(3)$, then all series $\sum_{k=1}^{\infty} g_{j}\left(w_{k}^{j}, w_{0}\right)$, $j=1, \ldots, n, w_{k}^{j}=\psi\left(z_{k}^{j}\right)$, converge as soon as $\Sigma_{1}^{\infty} g\left(w_{k}, w_{0}\right)<+\infty$. According to the equivalence of (1) and (2), and the well-known Blaschke theorem for the unit disc, we obtain the convergence of the following series: $\Sigma_{1}^{\infty}\left|w_{k}^{j}-t_{k}^{j}\right|, j=1, \ldots, n$. Here, $t_{k}^{j}$ is the closest point to $w_{k}^{j}$ on $\partial \mathcal{H C}$. It is clear that there exists a constant $M$ such that $\mid \omega_{j}\left(w^{\prime}\right)-$ $\omega_{j}\left(w^{\prime \prime}\right)|\leq M| w^{\prime}-w^{\prime \prime} \mid$ for all $w^{\prime}, w^{\prime \prime} \in \overline{\mathcal{H}}$. From this and according to the fact that all $\omega_{j}$ are invariant with respect to the conformal mappings, we obtain our statement. We note that in the last argument we only used the analyticity of $\partial \mathcal{K}$. Therefore, if $\partial G$ is analytic we obtain that (1)-(4) are equivalent to (5).

Let $a \in G, a^{\prime} \in \Gamma$. Following [3] we define the functions

$$
\begin{aligned}
& \mathscr{B}(z, a)=(z-a) \exp \left\{-\int_{\Gamma} \mathscr{P}(z, \zeta) \ln |\zeta-a| d \omega\left(E, z_{0}, G\right)\right\} \\
& \Re\left(z, a^{\prime}\right)=\left(z-a^{\prime}\right) \exp \left\{-\int_{\Gamma} \mathscr{P}(z, \zeta) \ln \left|\zeta-a^{\prime}\right| d \omega\left(E, z_{0}, G\right)\right\} .
\end{aligned}
$$

In the same way as it has been done in [3], one can show that the function (3.1) conformally maps $G$ onto the unit disc with slits along circular arcs centered at the origin. Similarly, the function (3.2) conformally maps $G$ onto the annulus with slits along circular arcs around the origin.

The proof of the following theorem is almost the same as that for the corresponding result in [3], so we omit it.

We recall that $\left\|q_{j k}\right\|_{1}^{n-1}=\left(\left\|\pi_{j k}\right\|_{1}^{n-1}\right)^{-1}$ (see $\left.\S 2\right)$.

THEOREM 3.2. Let the sequence $\left\{z_{k}\right\}_{1}^{\infty}$ satisfy (1)-(4) of Theorem 3.1. Let $\zeta_{k}^{j}=\psi^{-1}\left(t_{k}^{j}\right)$, where $t_{k}^{j}$ are the same as in the proof of Theorem 3.1 $(k=1, \ldots, ; j=1, \ldots, n)$. Then the product

$$
\mathscr{B}_{0}(z)=\prod_{j=1}^{n} \prod_{k=1}^{\infty} \frac{\mathscr{B}\left(z, z_{k}^{j}\right)}{\mathscr{B}\left(z, \zeta_{k}^{j}\right)}
$$

converges absolutely and uniformly on the compact subsets of $G . \mathscr{B}_{0}(z)=0$ if and only if $z \in\left\{z_{k}\right\}_{1}^{\infty}$. Moreover, if the sequence $\left\{G^{i}\right\}_{i=1}^{\infty}$ is the same as in $\S 1$, then $\left(\Gamma^{i}=\partial G^{i}=\bigcup_{k=1}^{n} \gamma_{k}^{i}\right)$

$$
\lim _{i \rightarrow \infty} \int_{\gamma_{k}^{\prime}}|\ln | \mathscr{\Im}_{0}(z)\left|-C_{k}\right| d \omega^{i}\left(E, z_{0}, G^{i}\right)=0
$$


where

$$
C_{k}=\sum_{i=1}^{\infty} \sum_{l=1}^{n} \sum_{j=1}^{n-1} q_{j k}\left(\delta_{j l}-\omega_{j}\left(z_{i}^{l}\right)\right), \quad k=1, \ldots, n-1 ; \quad C_{n}=0 .
$$

Finally, $\mid \Re_{0}(\zeta) \|_{\gamma_{k}}=\exp \left(C_{k}\right)$ a.e. with respect to $d \omega, k=1, \ldots, n$.

We shall call $\Re_{0}(z)$ the generalized Blaschke product.

For the reader's convenience let us recall the definitions of the basic classes of analytic functions in multiply connected domains. (Details can be found in $[\mathbf{1 0}]$.)

Let $f(z)$ be a single-valued analytic function in $G .\left\{G^{i}\right\}, \partial G^{i}=\Gamma^{i}$ are as above.

The function $f(z)$ belongs to $N(G)$ (Nevanlinna's class) if

$$
\varlimsup_{i \rightarrow \infty} \int_{\Gamma^{i}} \ln ^{+}|f| d \omega^{i}\left(E, z_{0}, G^{i}\right) \leq \mathrm{const}<+\infty .
$$

We say that $f(z)$ belongs to $N_{+}(G)$ if the integrals

$$
\left\{\int_{\Gamma^{i}} \ln ^{+}|f| d \omega^{i}\left(E, z_{0}, G^{i}\right)\right\}
$$

are uniformly absolutely continuous with respect to the harmonic measure. The function $f(z)$ belongs to $H_{p}(G), p>0$ (Hardy's classes), if

$$
\varlimsup_{i \rightarrow \infty} \int_{\Gamma^{i}}|f|^{p} d \omega^{i}\left(E, z_{0}, G^{i}\right) \leq \mathrm{const}<+\infty
$$

Finally, the function $f(z)$ belongs to $E_{p}(G), p>0$ (Smirnov's classes), if there exists the sequence $\left\{G^{i}\right\}$ as above such that $\Gamma^{i}=\partial G^{i}$ are rectifiable and

$$
\varlimsup_{i \rightarrow \infty} \int_{\Gamma^{i}}|f|^{p} d s \leq \mathrm{const}<+\infty
$$

It is known (see $[8-10,15,19])$ that $N \supset N_{+} \supset H_{p}$ and $N \supset E_{p}$ for all $p>0$.

COROLlaRY 3.1. If $f(z) \in N(G)$, then the Blaschke product corresponding to the zeroes of $f(z)$ converges. 
4. Factorization theorems in finitely connected domains. Let $G$ be an $n$-connected Jordan domain.

LEMMA 4.1. Let $f(z) \in N(G), f(z) \in N_{+}(G)$ or $f(z) \in H_{p}(G)$. Let $\Re_{0}(z)$ be the Blaschke product corresponding to the zeroes of $f(z)$. Then the function $\mathscr{F}(z)=f(z) / \mathscr{G}_{0}(z)$ belongs to the same class as $f(z)$.

Proof. For the classes $N, N_{+}$the lemma follows directly from the definitions and Theorem 3.1. Let $f(z) \in H_{p}(G)$. Then according to the results in [10], $f(z) \in N_{+}(G)$. So $\mathscr{F}(z) \in N_{+}(G)$. Moreover, the condition

$$
\int_{\Gamma}|\mathscr{F}|^{p} d \omega \leq \mathrm{const}<+\infty
$$

follows from the fact that

$$
\int_{\Gamma}|f|^{p} d \omega \leq \mathrm{const}<+\infty
$$

since $\mid B_{0}(\zeta) \|_{\gamma_{k}} \equiv$ const for all $k=1, \ldots, n$. Therefore from the generalization of the Polubarinova-Kochina theorem obtained in [10] it follows that $\mathscr{F}(z) \in H_{p}$. The lemma is proved.

The following theorem extends the result of R. Coifman and G. Weiss to Jordan domains.

THEOREM 4.1. Let $f(z) \in N(G)$. Then $f(z)$ can be represented in the following form:

$$
f(z)=Q(z) B_{0}(z) \exp \left\{\int_{\Gamma} \mathscr{P}(z, \zeta) d \mu(\zeta)\right\}
$$

where $B_{0}(z)$ is the Blaschke product corresponding to the zeroes of $f(z)$; $d \mu(\zeta)$ is a real Baire measure on $\Gamma$;

$$
Q(z)=\exp \left(\sum_{1}^{n-1} \lambda w_{j}(z)\right), \quad w_{j}(z)=\omega_{j}(z)+i \tilde{\omega}_{j}(z), j=1, \ldots, n-1,
$$

where $\lambda_{j}, j=1, \ldots, n-1$, are real numbers such that the numbers $(1 / 2 \pi) \Delta_{\gamma_{k}} \arg Q(z), k=1, \ldots, n$, are integers. The converse is also true, namely, if (4.1) holds with $\lambda_{j}, j=1, \ldots, n$, such that $(1 / 2 \pi) \Delta_{\gamma_{k}} \arg Q(z)$ are integers, then $f(z) \in N(G)$. 
Proof. Let $f(z) \in N(G)$, and let $B_{0}(z)$ be its Blaschke product. According to Lemma 4.1 $\mathscr{F}(z)=f(z) / B_{0}(z) \in N(G)$. Then, by Theorem $1.1, \ln |\mathscr{F}(z)|$ is representable in the form (1.2), i.e.

$$
\ln |\mathscr{F}(z)|=\int_{\Gamma} K(z, \zeta) d \mu(\zeta)
$$

where $d \mu$ is a real Baire measure on $\Gamma$. Consider the function $\int_{\Gamma} \mathscr{P}(z, \zeta) d \mu(\zeta)$. According to Theorem 2.1 we have

$$
u(z) \stackrel{\text { def }}{=} \operatorname{Re} \int_{\Gamma} \mathscr{P}(z, \zeta) d \mu=\ln |\mathscr{F}(z)|-\sum_{1}^{n-1} \lambda_{j} \omega_{j}(z) .
$$

Therefore,

$$
\mathscr{F}(z)=\exp \left(\sum_{1}^{n-1} \lambda_{j} w_{j}(z)\right) \exp \left\{\int_{\Gamma} \mathscr{P}(z, \zeta) d \mu(\zeta)\right\} .
$$

From this (4.1) follows. Since $\mathscr{F}(z)$ is single-valued, then

$$
(1 / 2 \pi) \Delta_{\gamma_{k}} \arg Q(z), \quad k=1, \ldots, n,
$$

are integers.

Assume (4.1) holds. Then the first two factors are bounded functions in $G$. Let us denote the third factor by $\phi(z)$. We have

$$
\begin{aligned}
\ln |\phi(z)| & =\int_{\Gamma} K(z, \zeta) d \mu(\zeta)-\sum_{1}^{n-1} \lambda_{j} \omega_{j}(z) \\
& =\int_{\Gamma} K(z, \zeta)\left[d \mu(\zeta)-\sum_{1}^{n-1} \lambda_{j} \omega_{j}(\zeta) d \omega\left(E, z_{0}, G\right)\right]
\end{aligned}
$$

So $\ln |\phi(z)|$ is representable by the Green-Stieltjes integral. Applying Theorem 1.1 we obtain that $\phi(z) \in N(G)$. Therefore, $f(z) \in N(G)$.

COROLlaRY 4.1. The factor $Q(z)$ can also be represented as

$$
Q(z)=\prod_{1}^{n-1}\left(B\left(z, a_{j}^{\prime}\right)\right)^{-m_{k}}
$$

where $a_{1}^{\prime}, \ldots, a_{n-1}^{\prime}$ are arbitrary points on $\lambda_{1}, \ldots, \lambda_{n-1}$, respectively, and $m_{k}$, $k=1, \ldots, n-1$, are integers defined by

$$
m_{k}=(1 / 2 \pi) \Delta_{\gamma_{k}} \arg \left[f(z) / B_{0}(z)\right] \text {. }
$$

Proof. Since $\mathscr{F}(z)=f(z) / B_{0}(z)$ is single-valued, then $m_{k}=$ $(1 / 2 \pi) \Delta_{\gamma_{k}} \arg \mathscr{F}(z), k=1, \ldots, n-1$, are integers. From (4.1) it follows 
that $\Delta_{\gamma_{k}} \arg \mathscr{F}=\Delta_{\gamma_{k}} \arg Q, k=1, \ldots, n-1$, i.e.

$$
m_{k}=\sum_{j=1}^{n-1} \pi_{k j} \lambda_{j}
$$

Hence, $\lambda_{j}=\sum_{k=1}^{n-1} q_{j k} m_{k}$. Fix arbitrary points $a_{k}^{\prime} \in \gamma_{k}, k=1, \ldots, n-1$. According to the result in [3], we have

$$
\left|B\left(z, a_{k}^{\prime}\right)\right|=\exp \left\{-\sum_{j=1}^{n-1} q_{j k} \omega_{j}(z)\right\}, \quad k=1, \ldots, n-1 .
$$

So

$$
\omega_{j}(z)=-\sum_{\nu=1}^{n-1} \pi_{j \nu} \ln \left|B\left(z, a^{\prime}\right)\right|
$$

Hence,

$$
\begin{aligned}
\sum_{1}^{n-1} \lambda_{j} \omega_{j}(z) & =-\sum_{k, \nu, j} m_{k} q_{k j} \pi_{j \nu} \ln \left|B\left(z, a^{\prime}\right)\right| \\
& =-\sum_{k, \nu} m_{k} \ln \left|B\left(z, a^{\prime}\right)\right| \sum_{j=1}^{n-1} q_{k j} \pi_{j \nu} \\
& =-\sum_{k=1}^{n-1} m_{k} \ln \left|B\left(z, a_{k}^{\prime}\right)\right| .
\end{aligned}
$$

From this we obtain our corollary.

THEOREM 4.2. $f(z) \in N_{+}(G)$ if and only if (4.1) holds with $d \mu=$ $\ln |f(\zeta)| d \omega\left(E, z_{0}, G\right)+d \nu$, where $f(\zeta)$ is the asymptotic boundary values of $f(z), d \nu \leq 0$ and $d \nu$ is singular with respect to $d \omega$.

Proof. Let $f(z) \in N_{+}(G)$. Then (4.1) holds with $d \mu$ taken from the representation of $\ln |\mathscr{F}(z)|$ by the Green-Stieltjes integral. Since, according to Lemma 4.1, $\mathscr{F}(z) \in N_{+}(G)$, then the integrals $\left\{\int_{\Gamma^{\prime}} \ln ^{+}|\mathscr{F}| d \omega^{i}\right\}$ are uniformly absolutely continuous with respect to the harmonic measure. Therefore, according to Theorem 1.5, we obtain

$$
\ln |\mathscr{F}(z)|=\int_{\Gamma} K(z, \zeta)\left[\ln |\mathscr{F}(\zeta)| d \omega\left(E, z_{0}, G\right)+d \nu\right]
$$

where $d \nu \leq 0$ and $d \nu$ is singular. But

$$
\ln |\mathscr{F}(\zeta)|=\ln |f(\zeta)|-\ln \left|B_{0}(\zeta)\right|=\ln |f(\zeta)|-C_{k}
$$


on $\gamma_{k}$. According to Theorem 2.1(2) we obtain

$$
\int_{\Gamma} \mathscr{P}(z, \zeta) \ln |f(\zeta)| d \omega=\int_{\Gamma} \mathscr{P}(z, \zeta) \ln |\mathscr{F}(\zeta)| d \omega
$$

Therefore, we can replace the measure $d \mu$ in (4.1) by the measure $d \mu^{\prime}=\ln |f(\zeta)| d \omega+d \nu$. The proof of the converse statement is the same as in Theorem 4.1.

THEOREM 4.3. $f(z) \in H_{p}(G)$ if and only if (4.1) holds with $d \mu=$ $\ln |f(\zeta)| d \omega+d \nu$, where $d \nu \leq 0$ and $d \nu$ is singular with respect to $d \omega$. Moreover,

$$
\int_{\Gamma}|f(\zeta)|^{p} d \omega\left(E, z_{0}, G\right) \leq \mathrm{const}<+\infty
$$

We omit the proof of this theorem since it can be obtained in the same way as the proofs of Theorems 4.1 and 4.2. The following theorem characterizes the Blaschke products (cf. $[8,9,15,19])$.

THEOREM 4.4. $f(z)$ is representable in the form $f(z)=Q(z) B_{0}(z)$, where $B_{0}(z)$ is the generalized Blaschke product, $Q(z)$ is the same as in Theorem 4.1, if and only if there exist real numbers $C_{1}, \ldots, C_{n}, C_{n}=0$, such that

$$
\lim _{i \rightarrow \infty} \int_{\gamma_{k}^{i}}|\ln | f\left|-C_{k}\right| d \omega^{i}\left(E, z_{0}, G^{i}\right)=0, \quad k=1, \ldots, n .
$$

Proof. If $f(z)=Q(z) B_{0}(z)$, then (4.2) follows from Theorem 3.2.

On the other hand, let us assume (4.2) holds. Then $f(z) \in N_{+}(G)$. Moreover, the integrals $\left\{\int_{\Gamma^{i}}|\ln | f|| d \omega\right\}$ are uniformly absolute continuous with respect to the harmonic measure. Hence, according to Theorem 1.2, $\ln |f(z)|$ is representable by the Green-Lebesgue integral. Therefore, the measure $d \mu$ in (4.1) is absolutely continuous with respect to $d \omega$. Moreover, on each $\gamma_{k}$ we have $\ln |f(\zeta)|=C_{k}$ a.e. From this and from Theorem 2.1(2) we obtain $f(z)=Q(z) \cdot B_{0}(z)$.

Let us now study the factorization problem for the classes $E_{p}$.

Let $G$ be a $n$-connected domain with the rectifiable Jordan boundary $\Gamma$. Hence, we can talk about ordinary angular boundary values. Moreover, in that case the singularity with respect to the harmonic measure and the singularity with respect to the Lebesgue measure $d s$ on $T$ are equivalent. 
We recall that $G$ is called a Smirnov domain $(G \in S)$ if $\ln \left|\psi^{\prime}(z)\right|$ is representable by the integral (1.3) (cf. [10]). (As above $\psi(z)$ is a conformal mapping of $G$ onto the circular domain $\mathscr{K}$.)

TheOrem 4.5. Let $G \in S$. Then $f(z) \in E_{p}(G), p>0$, if and only if (4.1) holds with $d \mu=\ln |f(\zeta)| d \omega+d \nu$, where $d \nu \leq 0, d \nu$ is singular and

$$
\int_{\Gamma}|f(\zeta)|^{p} d S \leq \text { const }<+\infty
$$

Proof. Since $G \in S, E_{p}(G) \subset N_{+}(G)$ for all $p>0$ (see [10]). So our theorem follows immediately from the Polubarinova-Kochina theorem in $[10]$.

To investigate the case $G \notin S$ we first recall the following generalization of the Keldysh-Lavrentjev theorem for multiply-connected domains proved in [10].

$$
f(z) \in E_{p}(G) \Leftrightarrow f(z) / \sqrt[p]{\psi^{\prime}(z)} \in H_{p}(G) .
$$

The following lemma is known (see [10]).

LemMA 4.2. Let $G_{j}, \gamma_{j} \subset \Gamma, \mathcal{H}_{j}, j=1, \ldots, n$, as above. Let $\psi_{j}(z)$ be a conformal mapping of $G_{j}$ onto $\mathcal{H}_{J}$. Then there exist constants $C_{1}, C_{2}$ such that the inequality

$$
0<C_{1} \leq\left|\psi_{j}^{\prime}(z) / \psi^{\prime}(z)\right| \leq C_{2}<+\infty
$$

holds near $\gamma_{j}$ for all $j=1, \ldots, n$.

From (4.3) it follows that $\psi^{\prime}(z) \in E_{1}(G)$. Moreover, since $\psi^{\prime}(z) \neq 0$ in $G$ we can write the representation (4.1) for $\psi^{\prime}(z)$ as

$$
\psi^{\prime}(z)=\exp \left\{\int_{\Gamma} \mathscr{P}(z, \zeta) d \mu_{0}\right\}
$$

where $d \mu_{0}=\ln \left|\psi^{\prime}(\zeta)\right| d \omega+d \nu_{0} ; d \nu_{0}$ is a singular measure. Since $1 / \psi^{\prime}(z)$ $=\left[\psi^{-1}(w)\right]^{\prime} \in H_{1}(\mathscr{K})(\Gamma$ is rectifiable!), from (4.3) and Theorem 4.5 it follows that $d \nu_{0} \geq 0$.

REMARK. We assume $\psi(z)$ maps the outer curve of $\Gamma$ onto the outer curve of $\partial K$. So $\ln \psi^{\prime}(z)$ is a single-valued function in $G$. 
THEOREM 4.6. $f(z) \in E_{p}(G), p>0$, if and only if (4.1) holds with $d \mu=\ln |f(\zeta)| d \omega+d \nu$, where $d \nu$ is singular, $d \nu \leq(1 / p) d \nu_{0}$ and $\int_{\Gamma}|f(\zeta)|^{p} d s<+\infty$

Proof. Let $f(z) \in E_{p}(G)$. Then, according to (4.1), we have

$$
f(z)=Q(z) B_{0}(z) \exp \left\{\int_{\Gamma} \mathscr{P}(z, \zeta) \ln |f(\zeta)| d \omega\right\} \exp \left\{\int_{\Gamma} \mathscr{P}(z, \zeta) d \nu\right\},
$$

where $d \nu$ is a singular measure. From (4.4) we obtain

$$
\begin{aligned}
\frac{f(z)}{\sqrt[p]{\psi^{\prime}(z)}}= & Q(z) B_{0}(z) \exp \left\{\int_{\Gamma} \mathscr{P}(z, \zeta) \ln \left|\frac{f(\zeta)}{\sqrt[p]{\psi^{\prime}(\zeta)}}\right| d \omega\right\} \\
& \times \exp \left\{\int_{\Gamma} \mathscr{P}(z, \zeta)\left[d \nu-\frac{1}{p} d \nu_{0}\right]\right\}
\end{aligned}
$$

Therefore, according to (4.3) and Theorem 4.3, we have $d \nu-(1 / p) d \nu_{0} \leq 0$ and

$$
\int_{\Gamma} \frac{|f(\zeta)|^{p}}{\left|\psi^{\prime}(\zeta)\right|} d \omega\left(E, z_{0}, G\right) \leq \mathrm{const}<+\infty
$$

So $d \nu \leq(1 / p) d \nu_{0}$. Since

$$
\left|\psi_{j}^{\prime}(\zeta)\right|=\partial g_{j}\left(\zeta, z_{0}\right) / \partial n_{\zeta}
$$

( $g_{j}$ is the Green function of $G_{j}$ ), then according to Lemmas 1.1 and 4.2 we obtain that (4.5) is equivalent to $\int_{\Gamma}|f(\zeta)|^{p} d s<+\infty$.

We omit a proof of the sufficiency since it is the same as in Theorems 4.1-4.3.

\section{Corollary 4.2. $\cap_{0<p<\infty} E_{p}(G) \subset N_{+}(G)$.}

Proof. Let $f(z) \in \bigcap_{0<p<\infty} E_{p}(G)$. Then according to Theorem 4.6 the singular part $d \nu$ of the measure $d \mu$ in the representation (4.1) for $f$ satisfies the inequality $d \nu \leq(1 / p) d \nu_{0}$ for all $p>0$. Letting $p$ tend to $\infty$ and using Theorem 4.2 we complete the proof.

Remark. From the results in [13] it is seen that the modulus of the boundary values and the singular measure are really the parameters of the corresponding classes $N, N_{+}, H_{p}$ and $E_{p}$. 


\section{REFERENCES}

[1] I. A. Alexandrov and A. S. Sorokin, The Schwarz problem in multiply-connected circular domains, (in Russian), Siberian Matem. J., XIII, No. 5, (1972), 971-1001.

[2] M. Brelot, On Topologies and Boundaries in Potential Theory, Springer-Verlag, 1971.

[3] R. Coifman and G. Weiss, A kernel associated with certain multiply-connected domains and its applications to factorization theoerms, Studia Math. XXVIII (1966), 31-68.

[4] E. A. Collingwood and A. J. Lohwater, The Theory of Cluster Sets, Cambridge University Press, 1960.

[5] L. E. Dunduchenko and S. A. Kas'yanyuk, On the subclasses of the Nevanlinna class of analytic functions in n-connected circular domains, (in Ukrainian), Dok. Akad. Nauk UkSSR, No. 9, (1959), 945-948.

[6] , Bounded analytic functions in multiply-connected domains (in Ukrainian), Dok. Akad. Nauk UkSSR, No. 2, (1959), 111-115.

[7] L. E. Dunduchenko, On the removing of multivaluedness of analytic functions in multiply-connected domains, (in Russian), Matem. Sb., 67 (109):1, (1965), 3-15.

[8] P. Duren, Theory of $H_{p}$ Spaces, Academic Press, New York, 1970.

[9] G. M. Goluzin, Geometric Theory of Functions of a Complex Variable, (Moscow 1952). English transl.: American Mathematical Society, Providence, Rhode Island, 1969.

[10] S. Ya. Havinson and G. C. Tumarkin, Classes of analytic functions in multiply-connected domains, In "Issledovaniya po sovremennym problemam teorii funkcii kompleksnogo peremennogo (Moscow 1960), pp. 45-77 (in Russian). French transl.: In "Fonctions d'une variable complexe. Problèmes contemporains", pp. 37-71; Gauthiers-Villars, Paris, 1962.

[11] On the representation of a harmonic function by the Green formula in $a$ multiply-connected domain, (in Russian), Matem. Sb., 44 (86):2, (1958), 225-234.

[12] S. Ya. Havinson, Removable sets of analytic functions of the Smirnov class (in Russian), in "Nekotorye voprosy sovremennoy teorii funkcii", ed. by P. P. Belinsky, Akad. Nauk SSSR, Sib. otd. ser. Matem., (1976), pp. 160-166.

[13] S. Ya. Havinson and G. C. Tumarkin, Existence of a single-valued function in a given class with a given modulus of its boundary values in multiply-connected domains, (in Russian), Izv. Akad. Nauk SSSR, Ser. Matem. 22, No. 4, pp. 3-52.

[14] D. Heighal, Classification theory for Hardy classes of analytic functions, Ann. Ac. Sci. Fenn., Ser. AI, 566, Helsinki, 1973.

[15] K. Hoffman, Banach Spaces of Analytic Functions, Prentice-Hall, Englewood Cliffs, 1962.

[16] P. I. Kuzhetčov and I. I. Privalov, Boundary value problems and certain harmonic and subharmonic functions in an arbitrary domain, Matem. Sb., 6 (48):3, (1939), 345-371.

[17] T. S. Kuzina, Parametrization of certain classes of analytic functions in multiply-connected domains, (in Russian), preprint.

[18] M. Parreau, Sur les moyennes des fonctions harmoniques et analytiques et la classification des surfaces de Riemann, Ann. Inst. Fourier, 3 (1951), 103-197.

[19] I. I. Privalov, Randeigenschaften Analytischer Funktionen, translated from the $2^{\mathrm{d}}$ Russian edition, Deutscher Verlag der Wissenschaften, Berlin, 1956.

[20] Certain classes of subharmonic functions and their analytic representations, (in Russian), Izv. Akad. Nauk SSSR, ser. Matem. No. 2, (1938), 131-220.

[21] S. Saks, Theory of the Integral, (2 revised edition), Monograf, Mat. 7, Warsaw, 1937. 
[22] P. M. Tamrazov, The generalization of the Blaschke product to multiply-connected domains (in Ukrainian), Dok. Akad. Nauk UkSSR, No. 7, (1962), 853-856.

[23] V. A. Zmorovič, The generalization of the Schwarz formula for multiply-connected domains, (in Ukrainian), Dok. Akad. Nauk UkSSR, No. 7, (1962), 853-856.

[24] E. I. Zverovič, Boundary value problems in the theory of analytic functions in Hölder classes on Riemann surfaces, (in Russian), Uspeky Mat. Nauk, 26 No. 1, (1971), 113-179.

Received May 30, 1980 and in revised form November 22, 1982.

BROWN UNIVERSITY

PROVIDENCE, RI 02912 


\title{
PACIFIC JOURNAL OF MATHEMATICS EDITORS
}

\author{
Donald BabBitT (Managing Editor) \\ University of California \\ Los Angeles, CA 90024 \\ Hugo Rossi \\ University of Utah \\ Salt Lake City, UT 84112 \\ C. C. Moore and Arthur Ogus \\ University of California \\ Berkeley, CA 94720
}

\author{
J. DugundiI \\ Department of Mathematics \\ University of Southern California \\ Los Angeles, CA 90089-1113 \\ R. FINN and H. SAMELSON \\ Stanford University \\ Stanford, CA 94305
}

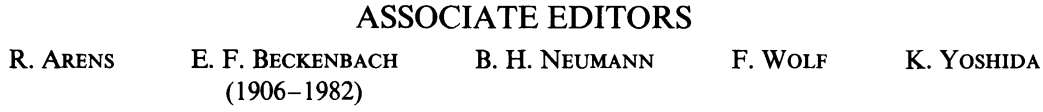

\section{SUPPORTING INSTITUTIONS}

UNIVERSITY OF ARIZONA
UNIVERSITY OF BRITISH COLUMBIA
CALIFORNIA INSTITUTE OF TECHNOLOGY
UNIVERSITY OF CALIFORNIA
MONTANA STATE UNIVERSITY
UNIVERSITY OF NEVADA, RENO
NEW MEXICO STATE UNIVERSITY
OREGON STATE UNIVERSITY

\author{
UNIVERSITY OF OREGON \\ UNIVERSITY OF SOUTHERN CALIFORNIA \\ STANFORD UNIVERSITY \\ UNIVERSITY OF HAWAII \\ UNIVERSITY OF TOKYO \\ UNIVERSITY OF UTAH \\ WASHINGTON STATE UNIVERSITY \\ UNIVERSITY OF WASHINGTON
}

The Supporting Institutions listed above contribute to the cost of publication of this Journal, but they are not owners or publishers and have no responsibility for its content or policies.

Mathematical papers intended for publication in the Pacific Journal of Mathematics should be in typed form or offset-reproduced (not dittoed), double spaced with large margins. Please do not use built up fractions in the text of the manuscript. However, you may use them in the displayed equations. Underline Greek letters in red, German in green, and script in blue. The first paragraph must be capable of being used separately as a synopsis of the entire paper. In particular it should contain no bibliographic references. Please propose a heading for the odd numbered pages of less than 35 characters. Manuscripts, in triplicate, may be sent to any one of the editors. Please classify according to the scheme of Math. Reviews, Index to Vol. 39. Supply name and address of author to whom proofs should be sent. All other communications should be addressed to the managing editor, or Elaine Barth, University of California, Los Angeles, California 90024.

There are page-charges associated with articles appearing in the Pacific Journal of Mathematics. These charges are expected to be paid by the author's University, Government Agency or Company. If the author or authors do not have access to such Institutional support these charges are waived. Single authors will receive 50 free reprints; joint authors will receive a total of 100 free reprints. Additional copies may be obtained at cost in multiples of 50 .

The Pacific Journal of Mathematics is issued monthly as of January 1966. Regular subscription rate: $\$ 132.00$ a year (6 Vol., 12 issues). Special rate: $\$ 66.00$ a year to individual members of supporting institutions.

Subscriptions, orders for numbers issued in the last three calendar years, and changes of address should be sent to Pacific Journal of Mathematics, P.O. Box 969, Carmel Valley, CA 93924, U.S.A. Old back numbers obtainable from Kraus Periodicals Co., Route 100, Millwood, NY 10546.

The Pacific Journal of Mathematics ISSN 0030-8730 is published monthly by the Pacific Journal of Mathematics at P.O. Box 969, Carmel Valley, CA 93924. Application to mail at Second-class postage rates is pending at Carmel Valley, California, and additional mailing offices. Postmaster: Send address changes to Pacific Journal of Mathematics, P. O. Box 969, Carmel Valley, CA 93924.

PUBLISHED BY PACIFIC JOURNAL OF MATHEMATICS, A NON-PROFIT CORPORATION

Copyright $(1983$ by Pacific Journal of Mathematics 


\section{Pacific Journal of Mathematics}

Vol. 108, No. $2 \quad$ April, 1983

Enrique Atencia and Francisco Javier Martin-Reyes, The maximal ergodic Hilbert transform with weights $\ldots \ldots \ldots \ldots \ldots \ldots \ldots \ldots \ldots . \ldots 257$

Bruce Blackadar, The regular representation of local affine motion

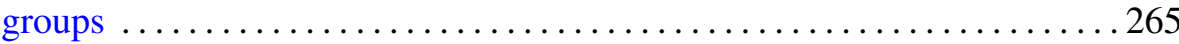

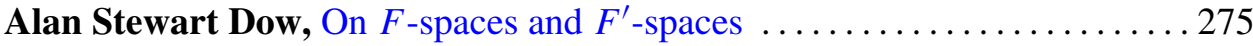

Yoshifumi Kato, On the vector fields on an algebraic homogeneous space . . 285

Dmitry Khavinson, Factorization theorems for different classes of analytic functions in multiply connected domains $\ldots \ldots \ldots \ldots \ldots \ldots \ldots \ldots 295$

Wei-Eihn Kuan, A note on primary powers of a prime ideal . . . . . . . . 319

Benjamin Michael Mann and Edward Yarnell Miller, Characteristic classes for spherical fibrations with fibre-preserving free group

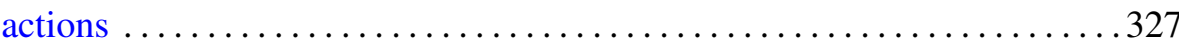

Steven Alan Pax, Appropriate cross-sectionally simple four-cells are flat . . . 379 R. K. Rai, On orthogonal completion of reduced rings ................ 385

V. Sree Hari Rao, On random solutions of Volterra-Fredholm integral

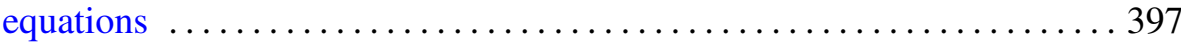

Takeyoshi Satō, Integral comparison theorems for relative Hardy spaces of solutions of the equations $\Delta u=P u$ on a Riemann surface $\ldots \ldots \ldots . .407$

Paul Sydney Selick, A reformulation of the Arf invariant one $\bmod p$ problem and applications to atomic spaces

Roelof Jacobus Stroeker, Reduction of elliptic curves over imaginary quadratic number fields

Jacob Towber, Natural transformations of tensor-products of representation-functors. I. Combinatorial preliminaries

James Chin-Sze Wong and Abdolhamid Riazi, Characterisations of amenable locally compact semigroups 